\title{
Translating Attentional Control Theory to Applied Psychological Eye Tracking Research
}

\author{
William R. Young \& Toby J. Ellmers
}

\begin{abstract}
This chapter will describe how eye tracking outcomes can be used to evaluate psychological processes, particularly in applied contexts (e.g., clinical anxiety disorders, elite motor performance and rehabilitation settings). Our discussion will focus primarily on the influence of emotion on two key aspects of attentional processing related to Attentional Control Theory: i) attentional bias for threatening stimuli, and ii) processing (in)efficiency. We present key metrics than can be used to infer these processes and describe that previous management of outcome measures can be allocated into two categories: transferred and retrofitted. This content is primarily aimed at readers thinking about using eye-tracking in the context of psychological research and practice; to help them design their task(s) and select appropriate outcome measures in order to avoid potential confounds.
\end{abstract}

\section{Background}

Unlike traditional paradigms evaluating attentional processes (e.g., dot-probe manual reaction-time tasks), eye tracking methodologies allow for more thorough examinations of the complex spatiotemporal aspects that underpin various attentional processes [1]. Recent advances in eye-tracking methodologies, including increased portability/head mounted systems, also permit eye-tracking in applied contexts and more dynamic tasks. Clinical practitioners and researchers are therefore increasingly turning to eye tracking to provide insight into the psychological mechanisms underpinning various clinical disorders, such as depression, autism and a range of anxiety disorders [2]. Eye tracking is also gaining greater interest in applied fields including sports [3,4], medical sciences [5,6], the military and law enforcement [7], aviation [8], driving [9] and civil engineering/hazard recognition $[10,11]$. However, unlike observations made in more reductionist tasks (e.g., the presentation of threatening/affective versus neutral words) - where outcome measures (and the corresponding underlying mechanisms they represent) are well-established [2] there are inherent difficulties of using eye-tracking to infer specific psychological processes in dynamic and complex (i.e., applied and ecologically valid) tasks. As a result, researchers in applied contexts have been largely preoccupied not with underlying attentional processes per se, but rather evaluating associations between eye-movement characteristics and performance outcomes; be it in perceptual, cognitive and/or motor domains. This approach is fundamentally limited for the simple 
reason that performance outcomes represent the culmination of many complex underlying factors, not just eye-movement characteristics. We argue that it is possible to use eye-tracking to infer both perceptual and attentional mechanisms that underpin behaviours that dictate performance outcomes. In this chapter, we attempt to conceptualise approaches taken across of range of fields of study using eye-tracking and provide a roadmap that articulates how key eye-tracking outcomes/metrics can be used by researchers to infer underlying psychological processes.

Researchers often use Attentional Control Theory [12] as a basis to explain the influence of anxiety and emotion on attentional processing, particularly with respect to cognitive and motor performance. ${ }^{1}$ According to Attentional Control Theory, the impact of anxiety on attention results from the disrupted balance between the goal-directed ("active" top-down attention influenced by prior experience and knowledge) and stimulus-driven attentional systems ("passive" bottom-up attention driven by salient and threatening stimuli) [13]. As a consequence, anxious individuals are less able to inhibit preferential attention from being directed towards either internal (e.g., worrisome thoughts) or external (e.g., an angry face in a crowd) threatening stimuli, and subsequently have difficulties diverting attention back away from these stimuli. Anxious individuals can use compensatory self-regulatory strategies to overcome these disruptions. Nonetheless, doing so is cognitively taxing, requires increased mental effort, and reduces the cognitive resources available for any other processes. Indeed, anxiety is argued to negatively impact processing efficiency - the level of cognitive resources needed to obtain a given level of performance - to a greater degree than it does performance effectiveness. Consequently, performance effectiveness is proposed to decline in situations whereby the anxious performer is unable to recruit sufficient compensatory cognitive resources.

We will use this chapter to argue that a variety of eye-movement outcome measures can be used to infer the key attentional processes implicated by Attentional Control Theory as being affected by anxiety and emotion; namely attentional bias for threats and processing (in)efficiencies. We will describe eye-tracking metrics that are commonly used within the context of anxiety and emotion, first in reductionist visual attention paradigms, and then in more applied research contexts.

The majority of reductionist research has focused on the influence of presenting emotional stimuli on temporal and spatial characteristics of visual attention. To a large extent, the focus on these aspects of visual attention is also reflected in applied studies; where researchers seek to better-

\footnotetext{
${ }^{1}$ In this context, attentional control refers to the cognitive ability to regulate the allocation of attention.
} 
understand the mechanisms underlying anxiety disorders and also the impact of emotion (often anxiety) on motor performance, whether it be a golfer making an important putt [14], a surgeon carrying out a life-altering precision task [15], or a fearful older adult walking on an uneven pavement $[16,17]$. Our discussion will focus on the influence of emotion on two key aspects of attentional processing related to Attentional Control Theory: 1) attentional bias for threatening stimuli, and 2) processing efficiency. We will discuss the emergent role of gaze tracking as a method to evaluate these psychological processes, particularly in applied contexts and complex environments. We describe a proposal that previous management of outcome measures can be allocated into two categories: transferred and retrofitted measures, and discuss rationale and issues associated with each approach. This content is primarily aimed at readers thinking about using eyetracking in their work; to help them design their task(s) and select appropriate outcome measures in order to avoid potential confounds. Using Attentional Control Theory ${ }^{2}$, we argue the importance of using a theoretical framework to inform both predictions and interpretations when attempting to infer attentional processes from observed eye movements. The attentional processes discussed in the sections below are listed in corresponding sections in Table 1.

\section{Attentional Bias for Threatening Stimuli}

The term attentional bias is used to describe the preferential direction of attention towards a salient stimulus [18]. Eye tracking methodologies are increasingly being used to examine attentional bias towards threatening (compared to neutral) stimuli, typically in clinical anxiety disorders and/or specific phobias [2].

\section{Vigilance bias}

Computer-based eye tracking paradigms have revealed consistent patterns of hypervigilant gaze behaviour in anxious individuals, whereby rapid initial fixations are directed towards threatening stimuli $[1,2,19-22]$. This evidence is largely based on observations of individual differences, often by comparing healthy controls with a group of participants with a clinically diagnosed anxiety disorder or self-reported phobia. For example, when passively viewing images of angry versus neutral faces, clinically anxious youths have been reported to make faster fixations towards angry (i.e., threatening) faces [1]. Anxious participants were also more likely to direct their initial fixation

\footnotetext{
${ }^{2}$ While we use Attentional Control Theory as the predominant theory governing the concepts described, it is important to acknowledge the important progressions made by more recent frameworks, such as the Integrated Model by Nieuwenhuys \& Oudejans [63,64]. We decided to focus on Attentional Control Theory within this chapter because its predictions relate to a broad range of attentional and cognitive factors, rather than conceptualising these factors as processes governing perceptual-motor performance.
} 
towards the threatening angry face. Rink and Becker [22] reported comparable patterns of results in spider phobics when viewing (and memorising) sequences of animal images. Phobic individuals were found to fixate images of spiders for significantly longer durations in the 500ms following stimulus presentation compared to non-anxious controls. These patterns of behaviours are termed vigilance bias [2] and likely reflect an automatic defensive response $[23,24]$ interpreted with respect to an evolutionary concept relating to the benefits of early threat detection [23]. The neuro-biological origins of this hypervigilance are suggested to reside in a pathway that is dedicated for the processing of threatening stimuli and operates in a 'bottom-up' fashion involving the retina, superior colliculus and amygdala (for review see [25]). These findings provide strong support for Attentional Control Theory's prediction that anxiety leads to increased dominance of the bottom-up stimulusdriven attentional system, leading to enhanced automatic processing of threatening stimuli.

Outcome measures used to infer early hypervigilance for threat (e.g., latency and location of initial fixation) have been employed to infer attentional bias towards threatening stimuli in a range of applied contexts. For example, during high-anxiety conditions, karate performers will fixate the attacking limbs of their opponent (e.g., the arm/fist) for longer durations [26]. Similar patterns of results have been reported during aiming tasks. For example, law enforcement officers performing a shooting exercise under conditions of low- (aiming at a mannequin target holding a handgun) and high-anxiety (aiming at an experienced firearms instructor who occasionally returned fire) exhibited fixations of increased durations towards threatening stimuli (i.e., the handgun) during high-anxiety conditions [27]. Footballers taking a penalty kick will also make significantly faster fixations towards the goalkeeper (the key threat in this task) during high-anxiety conditions [28]. When anxious, these participants also spent significantly longer fixating the goalkeeper compared to goal location when preparing to take the penalty kick (i.e., where they wished to kick the ball). Anxious novice drivers will also exhibit similar patterns of gaze behaviour when performing a driving simulator task under stressful conditions; increasing the number of fixations ahead towards other cars on the road (i.e., increased fixations towards potential collisions) at the expense of fixating the rear-view mirror [29]. Within the domain of perceptual-motor performance, such attentional biases are often associated with impaired performance (e.g., penalty shots placed closer to the goalkeeper and increased collisions with other cars) $[28,29]$. 


\begin{tabular}{|c|c|}
\hline Attentional process & Key outcome measures \\
\hline Attentional bias for threatening stimuli & \\
\hline i. Vigilance bias & $\begin{array}{l}\text { - } \\
\text { neurst fixation location (threatening versus } \\
\text { - Latency of first fixation towards threatening } \\
\text { stimuli }\end{array}$ \\
\hline ii. Maintenance versus avoidance bias & $\begin{array}{l}\text { - Number of fixations towards threatening } \\
\text { stimuli } \\
\text { Overall dwell time on threatening stimuli } \\
\text { (over both the trial itself, in addition to } \\
\text { 500ms time-bins) }\end{array}$ \\
\hline Processing (in)efficiency & $\begin{array}{l}\text { - } \quad \text { Search rate (task dependent) } \\
\text { - } \quad \text { Randomness (i.e., predictability) of visual } \\
\text { scanning (entropy metrics) } \\
\text { - } \quad \text { Latency to task-relevant fixation (for tasks } \\
\text { requiring the detection of a stimulus) } \\
\text { - } \quad \text { Total dwell time on task-relevant stimulus } \\
\text { prior to response (for tasks requiring a } \\
\text { decision) } \\
\text { - } \text { Fixations (number and duration) towards } \\
\text { peripheral movement cues }\end{array}$ \\
\hline
\end{tabular}

Table 1. Recommended eye-tracking outcomes used to assess two key aspects of attentional processing related to Attentional Control Theory: 1) attentional bias for threatening stimuli, and 2) processing (in)efficiency.

\section{Translating eye-movement metrics to applied contexts}

Whereas eye-movement metrics associated with early hypervigilance may appear to translate well between reductionist psychophysiological tasks and applied contexts, for the latter, there are several important issues that create complexity in the ability to interpret behaviours.

First, the generalisability of findings from an experimentally controlled paradigm to a real-world task will largely depend on the conditions for effective skill transfer; in other words, the degree of ecological validity concerning characteristics of both the dynamics of the task and environment in which it is performed. However, the implementation of this rich environmental and contextual information is likely to jeopardise the ability to maintain experimental control. Most importantly, 
any number of environmental features could place demands on attentional resources, the degree to which will depend on several factors such as visual saliency, task-relevance, if they are perceived as threatening, and their degree of stability (i.e., the expectation of whether the behaviour of the feature is predictable or requires constant monitoring). It is therefore important to consider and control as many of these factors as possible. When designing real world tasks these issues can pose significant (possibly unsurmountable) problems. Consequently, as described in the previous chapter, researchers are utilising new technologies (such as virtual reality) that afford the ability to control the sensory information available to participants, while still permitting the controlled assessment of eye-movements, even during dynamic and interactive tasks that contain a high degree of ecological validity. This degree of experimental control represents a major opportunity for researchers to isolate and study differences between specific participant/patient groups of interest and also changes that may occur over time (e.g. during training or rehabilitation).

Second, it is important to clearly define the most prominent threat in any given context and consider how interpretations of any related environmental feature may vary between participants. For example, a threatening stimulus for someone who is anxious about driving could be markedly different for an individual with a phobia of heights.

Third, whereas manual responses are now often removed from studies in the field of visual attention (in favour of eye-tracking methods), in the majority of applied contexts, it is the motor performance that ultimately determines task success. The inclusion of motor tasks (and corresponding evaluations of motor performance) into paradigms that also use eye movements to infer spatial and temporal characteristics of attention (e.g., hypervigilance for threat) may not readily appear to be problematic. However, researchers should consider the temporal sequencing between the presentation of any visual stimuli and the motor action to ensure that eye-movement metrics (e.g., saccadic latency to fixate a threatening stimulus) are not confounded by other eye-movements required to plan or execute the motor task. It is also likely that the concurrent performance of a motor task will carry attentional demands that could impact on saccadic latencies [30]. For example, a soccer player visually fixating a teammate to whom they plan to pass the ball may delay the transfer of visual fixation towards an oncoming defender (i.e., the threat). In this scenario, the attentional demands of the task of accurately passing the ball may impact on eye-movement latencies. 
The descriptions above are largely confined to metrics relating to early hypervigilant attentional responses to emerging threatening stimuli. In the following section we describe research documenting longer-latency responses.

\section{Maintenance vs avoidance bias}

While initial hypervigilance towards threatening stimuli appears to be a relatively robust finding across different tasks, threat stimuli and anxiety disorders (see [2] for a systematic review and metaanalysis), investigations into what gaze behaviour/s follow initial hypervigilance often present seemingly contradictory findings. Anxious individuals have been reported to display a maintenance bias [2], characterised by prolonged fixations towards threatening stimuli, i.e., increased overall dwell time. For example, veterans reporting higher symptoms of post-traumatic stress disorder (PTSD) fixated threatening, combat-related images during a free-viewing task for significantly longer durations than veterans in a low-PTSD group [31]. Similar patterns of results have also been reported in healthy, non-clinical populations performing a free-viewing task during the induction of state anxiety [32]. In contrast, other researchers have suggested that anxiety-related hypervigilance is followed by attentional avoidance [2] - particularly during extended viewing (e.g., >5 seconds). For example, despite displaying an initial hypervigilance towards spiders (as described above), spider phobics' subsequent fixations have been reported to occur further from the spider (compared to healthy controls) [33].

What is the reason for these seemingly contradictory findings? Armstrong and Olatunji [2] provided evidence to suggest that whether a threat leads to attentional maintenance versus avoidance may be dependent on the nature of the threat. For example, while PTSD is characterised by the maintenance of fixations towards combat-related threats, studies of spider phobia highlight eye avoidance gaze behaviours. Armstrong and Olatunji propose that threats related to combat pose a greater urgency and risk of immediate harm compared to spiders. In other words, a spider phobic may have learned that they can cope with the presence of spiders by looking away with little negative consequence (unless, of course, the spider is highly poisonous), whereas for someone with PTSD, disengaging from a threat during combat would likely have deadly consequences. The immediacy (and 'risk') of the threat seemingly plays a crucial mediating factor in whether fixations towards a threat is maintained or avoided. For example, in contrast to the avoidance behaviours observed in spider phobics when viewing photographs of spiders (e.g., [33]), spider phobics will maintain fixations towards live spiders during a 3-minute encounter [34]. Armstrong and Olatunji [2] propose that anxious individuals will engage in a cost-benefit analysis, whereby low-urgency threat 
cues (e.g., a photograph of a spider) are ignored in exchange for the reinforcement of reduced anxiety (i.e., avoidance behaviours). However, in contrast, these individuals would likely be motivated to continue visually monitoring immediate threats due to the heightened (perceived) risk for these to cause harm.

Maintenance and avoidance are believed to be mediated largely by attentional control [12,35]. As described in the Introduction, Attentional Control Theory [12] proposes that anxiety disrupts the balance between the bottom-up (stimulus-driven) and top-down (goal-directed) attentional systems. This leads to impaired attentional control, with anxious individuals less able to inhibit attention being directed towards threatening stimuli (either external threats or internal threats, e.g., worrisome thoughts) and subsequently find it harder to shift attention away from threatening stimuli. With increased effort (and if the cognitive resources are available), anxious individuals may be able to use compensatory strategies to overcome these disruptions (e.g., using conscious effort to actively disengage from, and thus avoid, a threatening stimulus). However, doing so is cognitively demanding and will impair processing efficiency. There is mounting evidence that these inefficiencies can be detected in eye-movements, especially during the planning phases of motor tasks. In the sections below, we describe previous observations to this effect. We then explain the origins of key emergent outcome measures used to infer processing (in)efficiencies in order to inform a subsequent discussion around the central issues that must be considered when designing eye-tracking studies in applied contexts.

\section{Processing Efficiency}

Processing efficiency is defined as the level of cognitive resources required to maintain effective task performance (i.e., response accuracy [12]), with efficiency decreasing as more resources are invested. While anxiety can impair task performance, it may be possible to maintain effective performance through investing additional effort (i.e., compensatory strategies) to counteract its negative, distracting effects. However, doing so reduces processing efficiency, as more cognitive resources are required to maintain the same level of performance. This is a central tenet of Attentional Control Theory [12]. Traditional methods to assess processing efficiency include selfreported measures of effort and dual-task paradigms [14]. However, researchers are increasingly using eye tracking to provide a more objective, within-task index of processing efficiency.

Before eye tracking can be used to assess processing efficiency, it is first necessary to determine what an efficient pattern of gaze behaviour looks like for a given task. Researchers commonly use 
visual search rates (the relationship between the number and duration of fixations) as an index of processing efficiency, with fewer fixations of longer durations thought to imply greater efficiency [14]. As saccades are believed to suppress visual processing [36], visual search strategies of fewer fixations of longer durations should therefore promote information extraction. Indeed, expertnovice comparisons highlight that expert performance in a range of domains (including surgery, lawenforcement, marksmanship, and sport) is characterised by lower search rates (i.e., small number of long-duration fixations towards pertinent visual cues [37]). This implies that experts will moreefficiently pick out the most important aspects of the visual scene required for successful task performance.

Eye-tracking research has demonstrated that anxiety decreases processing efficiency during a range of tasks and domains [14]. For example, Murray and Janelle [38] reported increased search rates (greater number of short-duration fixations) when completing a driving simulation task during highanxiety conditions, indicating that a greater number of fixations were required to acquire the same amount of perceptual information. One of the most robust findings from the literature in this area is that anxiety impairs processing efficiency as indexed through decreased gaze stability $[14,15,39-44]$. Effective performance in aiming tasks is consistently characterised by what is referred to as the 'quiet eye' [45]: a final task-relevant fixation lasting a minimum of 100 milliseconds that directly precedes initiation of movement. When performing a basketball free-throw under conditions of anxiety, Wilson, Vine and Wood [44] found that participants exhibited significantly shorter quiet eye periods, instead exhibiting a higher frequency of short duration fixations. These unstable gaze behaviours appeared to be directly related to decreased performance during the high-anxiety condition (i.e., significantly shorter quiet eye periods, and significantly higher number of short duration fixations, were exhibited during missed shots when anxious). Causer, Vickers, Snelgrove, Arsenault and Harvey [15] reported comparable patterns of results during a surgical task. Here, surgical residents once again exhibited quiet eye fixations of shorter durations when anxious, in conjunction with greater number of fixations towards other areas of interest during the task. Specifically, they were less likely to maintain a quiet eye fixation on the placement location, instead fixating the mechanics of the knot tying or hand motion. These inefficient visual search patterns were accompanied by less efficient (slower) hand movements and significant reductions in overall knot tying performance.

Rather than simply recording the number and duration of fixations, assessing the 'randomness' (i.e., predictability, as determined through metrics of entropy) of visual scanning may provide another 
useful index of processing efficiency. For example, Allsop and Gray [39] reported that pilots will exhibit more erratic patterns of visual search, transferring their gaze between different areas of interest in a less predictable manner, when completing a flight simulator task under conditions of heightened anxiety. In other words, the sequence of fixations between specific areas of interest became more difficult to predict; uncertainties that were correlated with greater increases in selfreported cognitive anxiety.

Further, not all tasks require the performer to maintain a stable fixation, for example, tasks in which the performer needs to search for, and identify, a certain stimulus. In these instances, processing inefficiencies are characterised by slower fixations towards the target stimulus (i.e. later fixation onset) - particularly during more difficult or challenging trials $[9,46]$. During more dynamic, sequential visuomotor tasks that require performers to use vision in a feed-forward manner to plan upcoming actions, reduced - rather than increased - search rates are used as an index of processing inefficiencies. For example, when traversing a climbing wall - a task that requires performers to scan ahead and select an appropriate path to follow - performers will display reduced search rates during a high-anxiety condition [47]. This implies that anxious performers required greater time to extract the same perceptual information acquired during baseline conditions. Similarly, when participants are required to identify and then respond to a particular stimulus on a computerised task, processing inefficiencies are characterised by increased dwell times on the stimulus prior to responding [48]. Finally, as peripheral vision can process movement-related information more efficiently than foveal vision [49], changes in the number and duration of fixations directed towards information typically processed with peripheral vision have also been used as a metric of processing inefficiency [14]. Indeed, increased anxiety has been shown to lead to performers relying on foveal, rather than peripheral, vision to guide movement and track the flight of a ball during a table tennis task [50]. It is therefore important to understand the various perceptual, cognitive and motor aspects of the task being performed, as these will determine markers of (in)efficiency that, in turn, can be used to inform meaningful assessment and interpretations.

\section{Transferred vs Retrofitted outcome measures}

A rich history of reductionist paradigms utilising pro- and anti-saccadic, forced choice and freeviewing tasks have given rise to established metrics deemed to provide a valid representation of hypervigilance. In simplified paradigms that minimise the amount/complexity of information available, it is reasonable to interpret eye-movement behaviours to infer spatial and temporal characteristics of attention. Some metrics have been transferred from reductionist to applied 
contexts as an equivalent measure. As described earlier in this chapter, this approach demands that, while the motor task may be dynamic, the i) visual scene, ii) any auditory or tactile information presented, and iii) the interactive nature of the task, is limited and/or carefully controlled to avoid confounds. In almost all applied contexts currently documented in the literature, limiting the task in this way fundamentally compromises its ecological validity and, therefore, the ability to translate findings to the true applied context.

Measures used to infer attentional processing of threatening stimuli have a strong precedent in reductionist paradigms. However, this rich literature is almost entirely derived from paradigms involving the presentation of a clearly defined threatening stimuli in the visual scene. However, in many applied contexts, the threat is not directly represented by a distinct feature/area of the visual scene. More commonly, a perceived threat will primarily relate to task failure, such as a surgeon tying a knot or a baseball batter facing a pitcher and oncoming ball. Therefore, when studying an applied context for the first time, there is insufficient theoretical precedent (i.e., that can be transferred from reductionist paradigms) to identify specific gaze behaviours that represent a given psychological construct. Instead, researchers start by describing observed behaviours and, through a process of associating these with performance outcomes or self-reported measures of attentional focus, retrofit a rationale for why a chosen metric represents a given psychological construct. Far from being a depreciative term, we argue that this process of retrofitting has been a necessity, as it allows the researcher to better understand how aspects of the task, environment and individual influence observed behaviour; which in turn provides the insight necessary to interpret behavioural characteristics in the context of psychological constructs and underlying theory. We must also appreciate that this approach of retrofitting helps to preserve the integrity and fidelity of the task being performed; ecological validity that would otherwise have been compromised through attempts to simplify the paradigm to a point where gaze outcomes could be transferred from reductionist literature. This approach also promotes the development of new outcome measures or contextualisation of eye-movement behaviours. For example, to our knowledge, the concept of quiet eye was born from observations of prolonged fixations in aiming tasks that were subsequently linked with increased shot success [51]. Only more recently have researchers started to explore the neural and psychological underpinnings of this concept.

The purpose of describing the origins of these metrics is two-fold. First, by acknowledging that most eye-tracking outcomes started out as simple behavioural descriptions, we might encourage researchers to interpret their descriptive observations to identify metrics that reflect a relevant 
psychological construct. This represents a critical step towards utilising underlying theoretical frameworks to form predictions; a process which, in turn, is critical for continued theoretical evolution. Second, the literature is at a point where several eye-tracking metrics have been associated with a given psychological construct (in this case, a broad construct of 'processing efficiency') and established across several contexts. We are therefore in a position to outline specific outcome measures likely to be most informative in the study of other applied contexts, depending on characteristics of the task and environment. These are listed in Table 1.

In summary, while hypervigilance is deemed to be characterised by rapid fixations towards threat across all contexts described, maintenance and processing efficiencies are far more dependent on the context in which they are assessed. If a task requires a stable gaze on a specific point (e.g., aiming at a target) then inefficiencies are characterised by saccades made to other areas, whereas if the task requires feedforward planning then efficient gaze behaviour may be characterised by multiple fixations towards the aspects of the environment that require movement planning. Alternatively, if the task is deemed to require the performer to process environmental features in their periphery, then foveation on that aspect of the environment might be characterised as inefficient.

It is important to state that the outcome measures listed in Table 1 should not be interpreted as 'valid' for use in any context. Rather, they are intended to provide a reference for researchers starting this process of identifying and retrofitting metrics that are best suited to their chosen context. In the following section we include a case study that gives examples of how the various outcome measures can be applied in a dynamic motor task.

\section{Case study: Adaptive gait in older adults}

Like many of the applied concepts discussed above, there are clear real-world applications for using the highlighted outcomes to study the way that people walk; particularly with respect to anxiety, e.g., fear of falling in older adults. Fear of falling is common in older adults and is independently associated with an increased risk of falls [52]. Identifying the specific mechanisms through which fear of falling can reduce safety in older adults is of therefore high importance. 


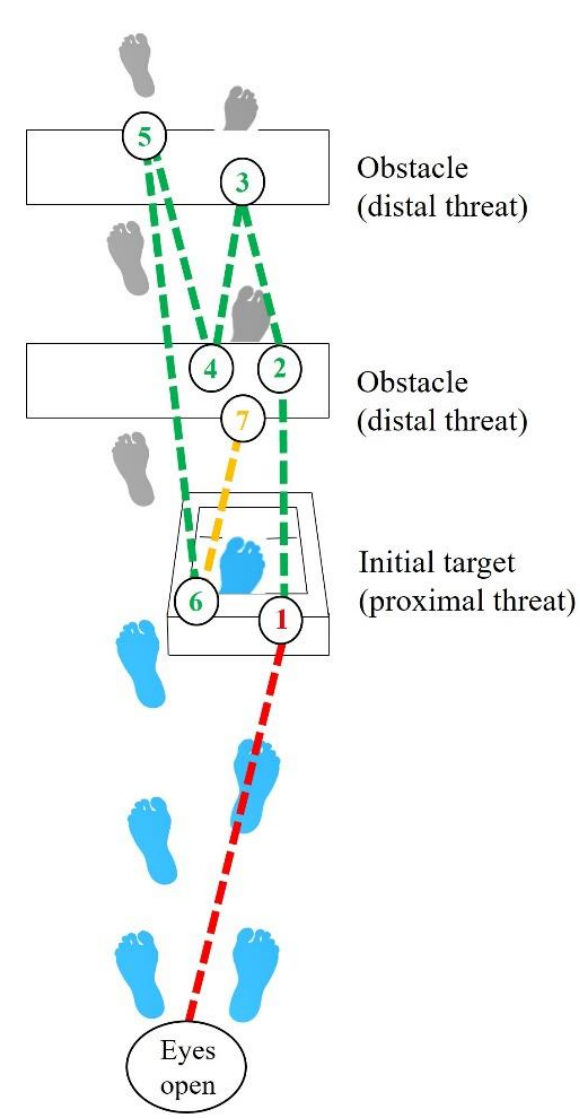

Figure 1. Example sequence of visual fixations during an adaptive gait task. The numbered sequence of eye-movements relate only to the approach to the first target (prior to foot contact), as represented by the blue footsteps. However, participants were required to complete the entire walking path. Participants also started every trial with their eyes closed until instructed to open them.

\begin{tabular}{|c|c|}
\hline $\begin{array}{l}\text { Fixation number } \\
\text { in Figure } 1\end{array}$ & Interpretation of outcome measure \\
\hline Eyes open -1 & $\begin{array}{l}\text { Hypervigilance to imminent threat (reduced latency of saccade provides } \\
\text { further inference of vigilance bias) - transferred }\end{array}$ \\
\hline $2-6$ & $\begin{array}{l}\text { Proactive visual search reflecting efficient feedforward planning - retrofitted } \\
\text { Absence of these eye-movements between distal threats/constraints and } \\
\text { corresponding maintenance of gaze fixation on the proximal threat infers } \\
\text { maintenance bias - transferred }\end{array}$ \\
\hline $6-7$ & $\begin{array}{l}\text { Interpretation depends on the temporal relationship with the time of foot } \\
\text { contact in the target. If foot contact occurs after the eye-movement then gaze } \\
\text { transfer from proximal to distal target is premature and reflects hypervigilance } \\
\text { - retrofitted }\end{array}$ \\
\hline
\end{tabular}


Table 2. Interpretations of eye-movements listed numerically in Figure 1

As we walk through our cluttered world, we build a visual-spatial map of our environment [53]. Proactive (or, 'feedforward') visual scanning of our walking environment allows not only for the identification of potential threats to balance, but also for the subsequent planning and execution of stepping behaviours necessary to avoid tripping [54]. While feedforward visual planning is critical for ensuring safety when walking, older adults also require on-line visual control to step safely (i.e., fixating a trip hazard during the step towards it to inform and modulate necessary adjustments to foot trajectory [55-57]). Our research highlights that anxiety can disrupt both feedforward and online visual control of locomotion through both an attentional bias for threatening stimuli and a reduction in processing efficiency.

In 2012, Young \& Hollands [58] reported observations of eye-gaze and stepping behaviours in an older adult (participant ' $\mathrm{P} 8$ ') both before and after a series of falls. She did not report any prior falls. During pre-fall observations, P8 displayed behaviours indicative of individuals deemed to be at a low risk of falling; proactively visually searching her intended walking path and previewing future stepping constraints. She exhibited these patterns of gaze while walking with a more upright posture and maintaining visual fixation on each stepping target until the step was completed inside it. When returning to the laboratory after the falls, P8 reported lower balance confidence and higher fear of falling. This was in the relative absence of any change in functional balance, visual or cognitive function. During the adaptive gait task, P8 displayed behaviour representative of anxious, 'high-risk' older adults (i.e., those deemed to be at a greater risk of falling due to impaired balance, muscle strength, etc.); walking with a stooped posture, looking down at her feet and only proximal areas of the walkway 1-2 steps ahead (i.e., very little proactive visual search). Her visual fixations were of a longer duration on the first target/imminent threat in her path (see Figure 1), and yet, she still looked away from the proximal target to the first distal threat (obstacle) before her step in the first target was complete. This 'early gaze transfer' was associated with increased stepping error in the target. These observations exemplify how behaviours commonly associated with frailty and fall-risk can be driven, at least in part, by increased anxiety about falling.

Table 3. Clinical example representing common behaviours observed in older adults. Note: some of the observations above represent unpublished data reported by Young \& Hollands [58]. 
We have investigated the above during a dynamic but semi-reductionist task, whereby threats to balance could be defined with relative clarity. In this task participants were required to approach and place one foot accurately inside a stepping target with raised edges, before continuing to step over two sequential obstacles (see Figure 1). Using both cross-sectional studies (i.e., comparing older adults with, and without, fear of falling [59]) and experimental manipulations (e.g., increased number of trip hazards [59], narrow raised walkway traversed without a safety harness $[17,60])$, our work has identified a consistent pattern of anxiety-related visual search behaviours during walking in young and older adults alike. As shown in Figure 1 (and corresponding Table 2), when anxious about falling, individuals will exhibit rapid [61] visual fixations towards more proximal stepping constraints (i.e., threats to balance) $[17,59,61]$. These behaviours indicate a hypervigilant bias for imminent threats to balance. During the approach to this stepping constraint/threat, anxious individuals will then display visual search behaviours indicative of processing inefficiencies. Specifically, they will exhibit restricted proactive visual scanning behaviours (i.e., reduced feedforward planning), characterised by both reduced search rates $[17,59,61]$ and decreased previewing fixations towards future stepping constraints (i.e., those beyond the initial constraint $[17,59,61]$ ). Unlike motor tasks that consist of a discrete aiming component (e.g., those described in the Processing Efficiency section above), gait requires vision to be used in a proactive, feedforward manner. Thus, reduced search rates and impaired visual planning is indicative of reduced processing efficiency - much in the same way as the previously described climbing example [47].

Our research provides evidence that these processing inefficiencies during gait are driven by both a maintenance bias (i.e., difficulties disengaging attention from the imminent threat to balance [59]) and cognitively demanding compensatory strategies, e.g., investing conscious effort into controlling each individual step (fixating 1-2 steps ahead to consciously regulate the ongoing step $[17,61]$ ). Once the anxious individual reaches the initial threat to their balance, this cycle of hypervigilant gaze behaviour will begin again; with preferential attention once again directed towards the next most immediate threat to their balance. For example, when stepping towards the initial constraint, anxious individuals will prematurely look away from the ongoing step (i.e., prior to the completion of the step) to fixate the subsequent threat to their balance $[59,61]$. It was originally believed that anxious individuals would prematurely direct their gaze towards future constraints due to having failed to acquire this information during the approach (i.e., indicative of a processing inefficiency [16]). However, our recent work has demonstrated that this 'early gaze transfer' behaviour is unrelated to reduced movement planning and instead reflects a hypervigilance for imminent threats 
to balance [61]. This gaze behaviour is causally related to increased stepping errors in older adults [62] and is a clear example through which anxiety can reduce safety while walking.

This case study illustrates that most of the visual search outcome described in the previous section to infer both attentional biases towards threats and processing inefficiencies can be readily translated to the applied context of gait (particularly, when fearful of falling). One exception to this is quiet eye which does not easily translate to the visual control of gait. First, unlike most of the motor tasks in which quiet eye has been studied (e.g., basketball free-throw, golf putting, surgical training, etc.), the 'target' during gait tasks tends to be the threat itself (e.g., trip hazard that needs to be safely avoided). It therefore becomes difficult to separate quiet eye fixations from fixations indicative of hypervigilance/maintenance bias. Second, unlike these other tasks, a step towards a target can be guided beyond the initiation of the movement, e.g., a basketball throw cannot be controlled once the movement has been initiated and the ball leaves the player's hands, whereas a step can be modified throughout the execution of the movement (i.e., during the swing phase of the step itself). This makes it difficult to identify the period in which to study quiet eye; given that this outcome is defined as a fixation of at least $100 \mathrm{~ms}$ directly preceding movement initiation [45].

The above descriptions relating to this case study demonstrate that the hypotheses presented in Attentional Control Theory [12] can be readily applied to the context of visual search behaviours during gait. The programme of research that we have conducted provides strong evidence that experiencing anxiety when walking will lead to an initial hypervigilance towards imminent threats to balance (i.e., faster fixations towards immediate stepping constraints). A maintenance bias will then be experienced (increased fixation dwell time on immediate constraints); however, compensatory strategies can be initiated in an attempt to reduce the negative effects of anxiety (i.e., fixations directed 1-2 steps ahead to consciously regulate each ongoing stepping movements). Irrespective, the outcome will be the same: reduced processing efficiency (as determined by restricted movement planning, e.g., reduction in search rate and the number of previewing fixations towards future/subsequent stepping constraints). Much like the cyclical nature of gait, these behaviours themselves also occur in a cyclical nature. Once the step towards the imminent threat to balance has been initiated, the cycle of hypervigilance begins anew and vision will once again be preferentially directed towards the next most immediate threat to balance.

\section{Conclusion}


This chapter highlights the unique opportunities offered by eye-tracking to infer attentional processes in applied contexts. Doing so, however, requires the selection of appropriate outcome measures (see Table 1 for a description of key outcomes used to infer attentional biases and processing efficiency). These outcomes can be categorised according to whether they have been transferred from reductionist paradigms or retrofitted (i.e., developed with consideration to the specific characteristics of a given task). We recommend that researchers adopt the appropriate (psychological) theoretical framework to enhance their ability to make informed interpretations. This will allow conclusions to be drawn that extend beyond the simply descriptive (e.g., documenting behaviours observed in a high-anxious patient group); providing the basis for researchers to draw inferences about underlying attentional processes driving these observations. While a number of challenges exist with respect to designing the appropriate task, and selecting suitable outcome measures, we hope that this chapter provides readers with a roadmap for using eye tracking methodologies in applied psychological research.

\section{Acknowledgements}

We would like to thank David Broadbent, David Harris and Sam Vine for their helpful feedback and comments on earlier drafts of this chapter.

\section{References}

1 Shechner T, Jarcho JM, Britton JC, et al. Attention bias of anxious youth during extended exposure of emotional face Pairs: An eye-tracking study. Depress Anxiety 2013;30:14-21. doi:10.1002/da.21986

2 Armstrong T, Olatunji BO. Eye tracking of attention in the affective disorders: A meta-analytic review and synthesis. Clin. Psychol. Rev. 2012;32:704-23. doi:10.1016/j.cpr.2012.09.004

3 Moran A, Campbell M, Ranieri D. Implications of eye tracking technology for applied sport psychology. J Sport Psychol Action 2018;9:249-59. doi:10.1080/21520704.2018.1511660

4 Payne KL, Wilson MR, Vine SJ. A systematic review of the anxiety-attention relationship in faraiming skills. Int Rev Sport Exerc Psychol 2019;12:325-55. doi:10.1080/1750984X.2018.1499796

5 Merali N, Veeramootoo D, Singh S. Eye-Tracking Technology in Surgical Training. J. Investig. Surg. 2019;32:587-93. doi:10.1080/08941939.2017.1404663 
Tien T, Pucher PH, Sodergren $\mathrm{MH}$, et al. Eye tracking for skills assessment and training: A systematic review. J. Surg. Res. 2014;191:169-78. doi:10.1016/j.jss.2014.04.032

Heusler B, Sutter C. Gaze Control and Training for High-Stress Situations in Law Enforcement: a Systematic Review. J. Police Crim. Psychol. 2020;35:401-13. doi:10.1007/s11896-01909338-1

8 PeißI S, Wickens CD, Baruah R. Eye-Tracking Measures in Aviation: A Selective Literature Review. Int J Aerosp Psychol 2018;28:98-112. doi:10.1080/24721840.2018.1514978

9 Wood G, Hartley G, Furley PA, et al. Working Memory Capacity, Visual Attention and Hazard Perception in Driving. J Appl Res Mem Cogn 2016;5:454-62. doi:10.1016/j.jarmac.2016.04.009 Jeelani I, Albert A, Han K, et al. Are Visual Search Patterns Predictive of Hazard Recognition Performance? Empirical Investigation Using Eye-Tracking Technology. J Constr Eng Manag 2019;145:04018115. doi:10.1061/(ASCE)CO.1943-7862.0001589

11 Han Y, Yin Z, Zhang J, et al. Eye-Tracking Experimental Study Investigating the Influence Factors of Construction Safety Hazard Recognition. J Constr Eng Manag 2020;146:04020091. doi:10.1061/(ASCE)CO.1943-7862.0001884

12 Eysenck MW, Derakshan N, Santos R, et al. Anxiety and cognitive performance: attentional control theory. Emotion 2007;7:336-53. doi:10.1037/1528-3542.7.2.336

13 Corbetta M, Shulman GL. Control of goal-directed and stimulus-driven attention in the brain. Nat Rev Neurosci 2002;3:201-15. doi:10.1038/nrn755

14 Wilson M. From processing efficiency to attentional control: a mechanistic account of the anxiety-performance relationship. Int Rev Sport Exerc Psychol 2008;1:184-201. doi:10.1080/17509840802400787

15 Causer J, Vickers JN, Snelgrove R, et al. Performing under pressure: Quiet eye training improves surgical knot-tying performance. Surg 2014;156:1089-96. doi:10.1016/j.surg.2014.05.004

16 Young WR, Mark Williams A. How fear of falling can increase fall-risk in older adults: Applying psychological theory to practical observations. Gait Posture 2015;41:7-12. doi:10.1016/j.gaitpost.2014.09.006

17 Ellmers TJ, Cocks AJ, Young WR. Evidence of a Link Between Fall-Related Anxiety and High- 
Risk Patterns of Visual Search in Older Adults During Adaptive Locomotion. Journals Gerontol Ser A 2020;75:961-7. doi:10.1093/gerona/glz176

Crombez G, Van Ryckeghem DML, Eccleston C, et al. Attentional bias to pain-related information: A meta-analysis. Pain 2013;154:497-510. doi:10.1016/j.pain.2012.11.013

19 Gamble AL, Rapee RM. The time-course of attention to emotional faces in social phobia. J Behav Ther Exp Psychiatry 2010;41:39-44. doi:10.1016/j.jbtep.2009.08.008

Nelson AL, Purdon C, Quigley L, et al. Distinguishing the roles of trait and state anxiety on the nature of anxiety-related attentional biases to threat using a free viewing eye movement paradigm. Cogn Emot 2015;29:504-26. doi:10.1080/02699931.2014.922460

21 Thomas CL, Goegan LD, Newman KR, et al. Attention to threat images in individuals with clinical and subthreshold symptoms of post-traumatic stress disorder. J Anxiety Disord 2013;27:447-55. doi:10.1016/j.janxdis.2013.05.005

22 Rinck M, Becker ES. Spider fearful individuals attend to threat, then quickly avoid it: Evidence from eye movements. J Abnorm Psychol 2006;115:231-8. doi:10.1037/0021-843X.115.2.231

23 LeDoux JE. Coming to terms with fear. Proc. Natl. Acad. Sci. U. S. A. 2014;111:2871-8. doi:10.1073/pnas.1400335111

24 LeDoux JE, Pine DS. Using neuroscience to help understand fear and anxiety: A two-system framework. Am. J. Psychiatry. 2016;173:1083-93. doi:10.1176/appi.ajp.2016.16030353

25 Mulckhuyse $M$. The influence of emotional stimuli on the oculomotor system: A review of the literature. Cogn. Affect. Behav. Neurosci. 2018;18:411-25. doi:10.3758/s13415-018-0590-8

26 Williams AM, Elliott D. Anxiety, expertise, and visual search strategy in karate. J Sport Exerc Psychol 1999;21:362-75. doi:10.1123/jsep.21.4.362

27 Nieuwenhuys A, Oudejans RRD. Training with anxiety: Short-and long-term effects on police officers' shooting behavior under pressure. Cogn Process 2011;12:277-88. doi:10.1007/s10339-011-0396-x

28 Wilson MR, Wood G, Vine SJ. Anxiety, attentional control, and performance impairment in penalty kicks. J Sport Exerc Psychol 2009;31:761-75. doi:10.1123/jsep.31.6.761

29 Gotardi GC, Polastri PF, Schor P, et al. Adverse effects of anxiety on attentional control differ as a function of experience: A simulated driving study. Appl Ergon 2019;74:41-7. doi:10.1016/j.apergo.2018.08.009 

eye movements. Acta Psychol 2000;104:69-85. doi:10.1016/S0001-6918(99)00054-2 veterans of the Iraq war. J Anxiety Disord 2010;24:293-9. doi:10.1016/j.janxdis.2009.12.006

32 Quigley L, Nelson AL, Carriere J, et al. The effects of trait and state anxiety on attention to emotional images: An eye-tracking study. Cogn Emot 2012;26:1390-411. doi:10.1080/02699931.2012.662892 Pflugshaupt T, Mosimann UP, Von Wartburg R, et al. Hypervigilance-avoidance pattern in spider phobia. J Anxiety Disord 2005;19:105-16. doi:10.1016/j.janxdis.2003.12.002 Lange WGT, Tierney KJ, Reinhardt-Rutland AH, et al. Viewing behaviour of spider phobics and non-phobics in the presence of threat and safety stimuli. Br J Clin Psychol 2004;43:235-43. doi:10.1348/0144665031752989

35 Cisler JM, Koster EHW. Mechanisms of attentional biases towards threat in anxiety disorders: An integrative review. Clin. Psychol. Rev. 2010;30:203-16. doi:10.1016/j.cpr.2009.11.003 Bridgeman B, Hendry D, Stark L. Failure to detect displacement of the visual world during saccadic eye movements. Vision Res 1975;15:719-22. doi:10.1016/0042-6989(75)90290-4

37 Mann DY, Williams AM, Ward P, et al. Perceptual-cognitive expertise in sport: A metaanalysis. J Sport Exerc Psychol 2007;29:457-78. doi:10.1123/jsep.29.4.457

38 Murray NP, Janelle CM. Anxiety and performance: A visual search examination of the processing efficiency theory. J Sport Exerc Psychol 2003;25:171-87. doi:10.1123/jsep.25.2.171 Allsop J, Gray R. Flying under pressure: Effects of anxiety on attention and gaze behavior in aviation. J Appl Res Mem Cogn 2014;3:63-71. doi:10.1016/j.jarmac.2014.04.010 Behan M, Wilson M. State anxiety and visual attention: The role of the quiet eye period in aiming to a far target. J Sports Sci 2008;26:207-15. doi:10.1080/02640410701446919

41 Causer J, Holmes PS, Smith NC, et al. Anxiety, Movement Kinematics, and Visual Attention in Elite-Level Performers. Emotion 2011;11:595-602. doi:10.1037/a0023225

42 Nibbeling N, Oudejans RRD, Daanen HAM. Effects of anxiety, a cognitive secondary task, and expertise on gaze behavior and performance in a far aiming task. Psychol Sport Exerc 2012;13:427-35. doi:10.1016/j.psychsport.2012.02.002 

behaviours vary depending on expertise and anxiety levels in ten-pin bowling. J Sports Sci 2018;36:2076-86. doi:10.1080/02640414.2018.1436764

44 Wilson MR, Vine SJ, Wood G. The influence of anxiety on visual attentional control in basketball free throw shooting. J Sport Exerc Psychol 2009;31:152-68. doi:10.1123/jsep.31.2.152

45 Vickers J. Perception, Cognition, and Decision Training: The Quiet Eye in Action. Champaign, IL: Human Kinetics 2007.

Smith AR, Haller SP, Haas SA, et al. Emotional distractors and attentional control in anxious youth: eye tracking and fMRI data. Cogn Emot 2020; Advance online publication. doi:10.1080/02699931.2020.1816911

47 Nieuwenhuys A, Pijpers JR, Oudejans RRD, et al. The influence of anxiety on visual attention in climbing. J Sport Exerc Psychol 2008;30:171-85.

48 Derakshan N, Koster EHW. Processing efficiency in anxiety: Evidence from eye-movements during visual search. Behav Res Ther 2010;48:1180-5. doi:10.1016/j.brat.2010.08.009

49 Milner D, Goodale M. The Visual Brain in Action. Oxford: : Oxford University Press 1995. doi:10.1093/acprof:0so/9780198524724.001.0001

50 Williams AM, Vickers J, Rodrigues S. The Effects of Anxiety on Visual Search, Movement Kinematics, and Performance in Table Tennis: A Test of Eysenck and Calvo's Processing Efficiency Theory. J Sport Exerc Psychol 2001;23:438-55. doi:10.1123/jsep.23.4.438

51 Vickers JN. Gaze control in putting. Perception 1992;21:117-32. doi:10.1068/p210117

52 Friedman SM, Munoz B, West SK, et al. Falls and Fear of Falling : Which Comes First ? A Longitudinal Secondary Prevention. J Am Geriatr Soc 2002;50:1329-35. doi:10.1046/j.15325415.2002.50352.x

53 Zettel JL, Scovil CY, Mcllroy WE, et al. Gaze behavior governing balance recovery in an unfamiliar and complex environment. Neurosci Lett 2007;422:207-12. doi:10.1016/j.neulet.2007.06.020

54 Matthis JS, Barton SL, Fajen BR. The critical phase for visual control of human walking over complex terrain. Proc Natl Acad Sci 2017;114:E6720-9. doi:10.1073/pnas.1611699114 
in the travel path? Neuroreport 1997;8:3661-5.

Chapman GJ, Hollands MA. Age-related differences in stepping performance during step cycle-related removal of vision. Exp brain Res 2006;174:613-21. doi:10.1007/s00221-0060507-6

57 Chapman GJ, Hollands MA. Evidence that older adult fallers prioritise the planning of future stepping actions over the accurate execution of ongoing steps during complex locomotor tasks. Gait Posture 2007;26:59-67. doi:10.1016/j.gaitpost.2006.07.010

58 Young WR, Hollands MA. Newly Acquired Fear of Falling Leads to Altered Eye Movement Patterns and Reduced Stepping Safety: A Case Study. PLoS One 2012;7. doi:10.1371/journal.pone.0049765

Young WR, Wing AM, Hollands MA. Influences of state anxiety on gaze behavior and stepping accuracy in older adults during adaptive locomotion. Journals Gerontol - Ser B Psychol Sci Soc Sci 2012;67 B:43-51. doi:10.1093/geronb/gbr074

60 Ellmers TJ, Young WR. The influence of anxiety and attentional focus on visual search during adaptive gait. J Exp Psychol Hum Percept Perform 2019;45:697-714.

http://dx.doi.org/10.1037/xhp0000615

61 Ellmers TJ, Young WR. The influence of anxiety and attentional focus on visual search during adaptive gait. J Exp Psychol Hum Percept Perform 2019;45:697-714. doi:10.1037/xhp0000615

62 Young WR, Hollands MA. Can telling older adults where to look reduce falls? Evidence for a causal link between inappropriate visual sampling and suboptimal stepping performance. Exp Brain Res 2010;204:103-13. doi:10.1007/s00221-010-2300-9

63 Nieuwenhuys A, Oudejans RRD. Anxiety and perceptual-motor performance: Toward an integrated model of concepts, mechanisms, and processes. Psychol Res 2012;76:747-59. doi:10.1007/s00426-011-0384-x

64 Nieuwenhuys A, Oudejans RR. Anxiety and performance: perceptual-motor behavior in highpressure contexts. Curr Opin Psychol 2017;16:28-33. doi:10.1016/j.copsyc.2017.03.019 